\title{
Optical Mark Recognition using Open CV
}

\author{
Pooja Raundale, $\mathrm{PhD}$ \\ H. O. D, MCA \\ Department, \\ Sardar Patel Institute of \\ Technology \\ Mumbai, India
}

\author{
Taruna Sharma \\ Professor, MCA \\ Department, \\ Sardar Patel Institute \\ of Technology \\ Mumbai, India
}

\author{
Saurabh Jadhav \\ Student, MCA \\ Department, \\ Sardar Patel Institute \\ of Technology \\ Mumbai, India
}

\author{
Rajan Margaye \\ Student, MCA \\ Department, \\ Sardar Patel Institute \\ of Technology \\ Mumbai, India
}

\begin{abstract}
The concept behind this paper is to develop a system that can check and evaluate the MCQ answers using the webcam. Till date many institution and organization held many exams where user are provided with a separate question and answer sheet, where answer are multiple choice option, each option may be a square or circle and user is supposed to either tick or fill it using pen or pencil. Such exams are JEE ,CET, NEET etc. Also many financial institution and college refer such types of test since MCQ format Q\&A are efficient and time saving as compared to that of lengthy papers. The renowned high level organization or institution use OMR technology for assessment of such paper, since number of student are in million and it's not feasible to check each single paper as it is time consuming, also it requires lot of human labor another problem is that human are prone to make error for example humans may grade wrong answer as correct / correct answer as wrong, Human may be biased about particular student and may score him less mark.
\end{abstract}

Maintaining paper for longer time is troublesome since it is prone to various climate change (fire, moisture etc) also humans have to manually record all the scores in different excel sheet/ file. On top of that high level organization use huge scanner which scans each paper in continuous batch precisely, where as normal school or college cannot afford such type of machine . Here the aim was to build a system such that when answer sheet is placed before webcam it will automatically get its image and all the answer will be evaluated and result will be displayed, determining score and total percentage. Since laptop is easily available to all, this make system more easy to handle and accessible to all as compared to other systems.

The problem statement is divided into 2 phases, First is how to extract image using 'open cv' and Second is how to apply content-filteration and image processing algorithm on it to get expected result.

\section{Keywords}

Optical Mark Recognition

\section{INTRODUCTION}

Conducting multiple choice questions examination worldwide has become one of the common means for evaluation of students credibility. Not only in India but also in other country various examinations are held which are in format of MCQ, In india JEE MAINS (Joint Entrance Exam) held by IIT, AIPMT (All Indian Pre-Medical Entrance Test) held by central board of ministry education, MHT-CET are such of the important exams that use this format to conduct examination. Also worldwide exams like TOEFL and GRE, GMAT are MCQ based. Reason for conducting MCQ exams is, it saves times in terms of time and evaluation, which makes it one of the efficient approach to conduct exams.
Every year millions of student appear for this test/ exam, where they read each question and they are supposed to mark or highlight there answer using pen or pencil. Currently high level organization that conduct exams like JEE, NEET have high configuration machine like special scanner which is costly, where this scanner are highly capable of capturing images and performs evaluation on further basis, but normal college cannot afford it. At present OMR technology is very expensive, not only because of the hardware requirement but also because of OMR software, as it's obvious that software that reduces the human effort and error paves the way to extra expense. Hence small school, institute cannot use this technology if they want to conduct normal test because of above challenges.

They resort to manually check the paper and note down all the answer of particular student and determine whether student has qualified or not. On an average based on number of question's evaluating each paper may take around $5 \mathrm{~min}$. Hence if there are 100 students then it will take around 500 minutes or 8.3 hours of evaluation time which is not acceptable. The main purpose of our project was to save money as well as time and manpower, which is spent more by traditional approach, to overcome it. It's obvious that the OMR we are proposing does not match the efficiency and performance of high level OMR used by various institutes but it fulfill most of the need of various institution and organization and solves above problem. Now a days laptop are easily available and are one of the common mode of equipment used by all institutions, hence we thought that using laptop's webcam would be efficient approach to use for OMR. As webcam is builtin hardware and does not incur extra cost for installation of driver.

Here proposed OMR does following steps:

$\square \quad$ Capture answer sheet image using webcam.

$\square \quad$ Perform extraction of image page.

$\square \quad$ Content filtration and apply image processing algorithm.

$\square \quad$ Evaluate which answer is correct or incorrect and display total marks.

$\square \quad$ After evaluation generate excel sheet and record all marks in it.

Below is image of answer sheet that will be used to evaluate 


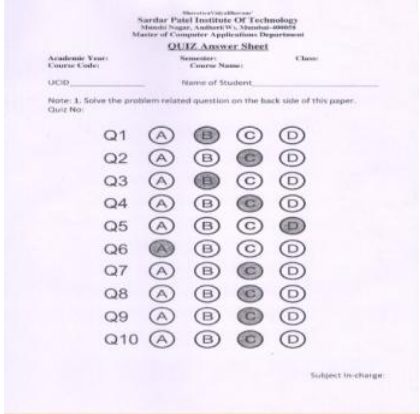

Fig. 1 Answer Sheet

Here the algorithm is made in such a way that it know that the answer sheet is rectangular in shape in predefined text format, has fixed number of options for each question, with appropriate size and shape of each options.

\section{APPLICATION FLOW}

A) Image capturing: First thing that needs to be done is to turn on webcam of laptop, after webcam is turned on next thing that need to be done is place the answer sheet in front of webcam and then press space button in order to capture image. Note during doing this task it's necessary that the answer sheet is placed properly in front of webcam and it does not reflect any shadow on page since it may affect content filtration task, later will discuss in future why shadow may be huge concern during performing this task.

B) Content Filtration: After getting screenshot image, next task is to extract necessary part of image since we don't need entire part of image. We draw a rectangle on\ image and image inside that rectangle will get extracted. This image extracted will further be used for image processing, which acts as input.

C) Image processing: After image has been provided as input to this module of algorithm, then next step is to perform some sort of pre processing on it and then apply actual image processing algorithm on it. Here next step is to provide actual answer to our algorithm and after performing transformation on image it will check whether answer matches the actual one or not, and based on it will show how many are correct and how many are wrong and show percentage acquired. Here if answer is correct then it will highlight it with green border and if wrong then we will highlight it with red border.

D) Storing Result: In this stage marks will be automatically be stored in excel file after each answer paper gets checked.

\section{ALGORITHM AND IMPLEMENTATION}

This stage will discuss detailed working of system, and will cover basic intuition of how system is actually working.

Step 1: Here take snapshot of answer sheet using webcam for this, It needs VideoCapture and NamedWindow function of opencv. Further when the paper is held in front of webcam then press button to take screenshot. This can be achieved using NamedWindow that check whether desired button is clicked by check its event's ascii code if yes then capture the frame of webcam and then write it to desired location.
Step 2: Next step is that there is no need of entire image. Only the answer sheet with little background to it is needed, hence one needs to crop the image. Here one may need opencv and key events.

When left button is clicked and its position changes there is need to store all of its coordinates, after the button releases select the area of that coordinates that form rectangle and crop that particular part and while discarding the remaining other parts. This way the input for OMR system is generated.

Step 3: After having the filtered image, the next step will be to convert the image in grayscale format, since it will be helpful for easily detecting marked/ highlighted done by user. Later image will undergo high blur so that we can reduce high frequency noise, that means distortion of image.

Step 4: After converting to grayscale, next step will be to extract page from the given image. For this apply Canny Edge Detection technique it helps to find all edges within image, This algorithm is multi stage algorithm which goes through various steps and produces image which has all edges (horizontal and vertical) within it.

\section{Start}
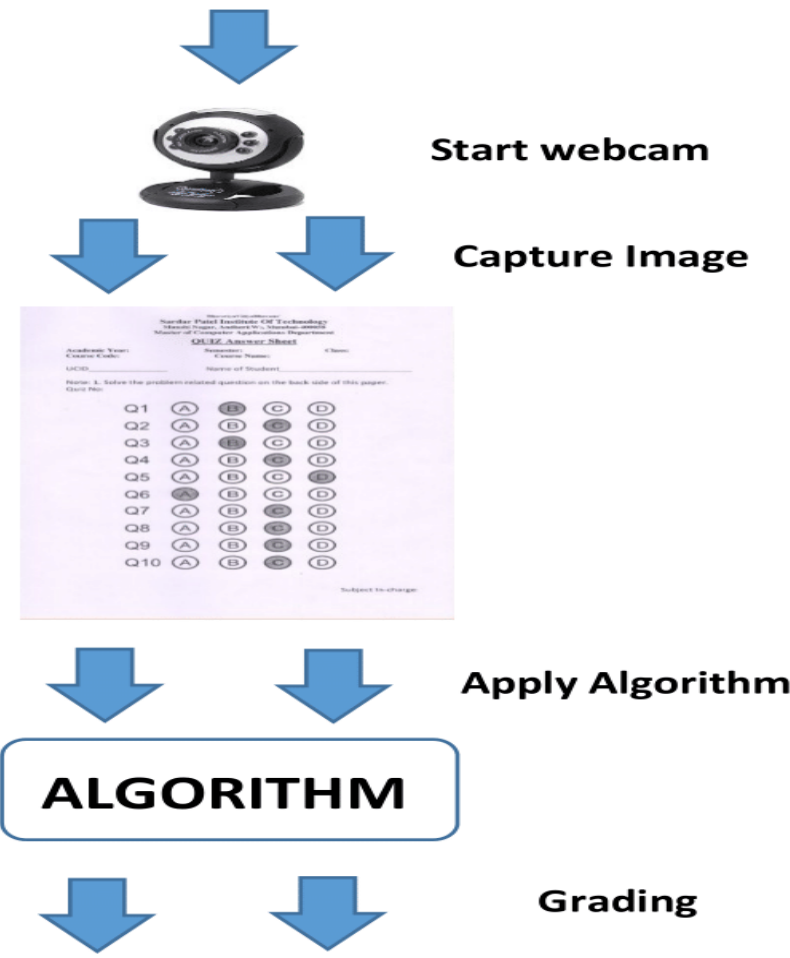

Grading

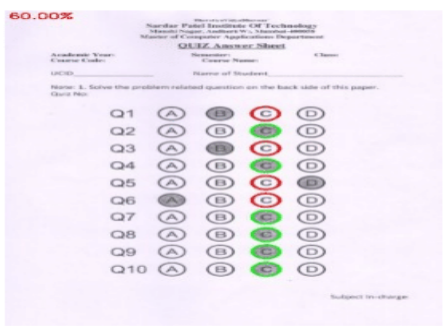

\section{Application Flow}

Fig. 2 
Step 5: After getting outline, Apply transformation such that it give top-down, bird's eye view of previous image. This is done in order to get four border points of our exam paper. This find out this four points using open cv library "cv.findContours()". Here it's mandatory to apply Canny Edge Detection before apply this step. As in this stage the aim is to search for white structure from black background and such transformation is only possible using previous step. In order to get only corner border points rather than a continuous line that form's a rectangle (page structure) pass parameter "cv.CHAIN_APPROX_SIMPLE", It only extract corner point, thus removing redundant points.

Step 6: After getting contours, sort them, so that higher contour are in front of our array and small contour are at last, the basic intuition behind this logic is that the 4 border contour extracted are larger than the contour of object present within the image. Here answer sheet image should be main focal point within the entire image.

Step 7: In order to achieve top-down bird eye view use "four_point transform()" function. This function is used for geometric transformation to extract only page image from entire image. There may be need to try various approach of getPerspectiveTransform() in order to get best suited result. The reason behind performing perspectiveTransform is to get 90 degree view of our image.

Step 8: Later do binarization of image in short converting image upto 256 gray level to black and white. It is used as pre processing before any OCR or OMR logic applies. This can be done using Otsu's transformation. As one can see below is generated black and white image, this binarization is done using threshold() of open cv and passing otsu parameter to it.

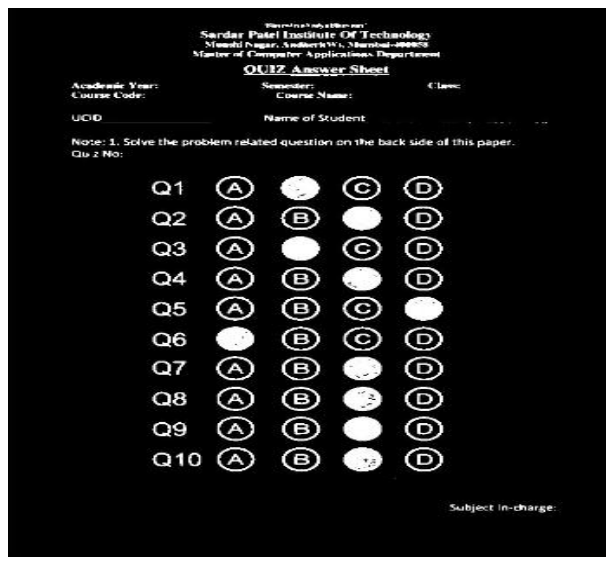

Fig. 3 Gray scale image

Step 9: Next step is to extract bubble options present within the answer page for this we again use perspective transformation and binarization, In order to find bubbles within image there are many ways, Here use HoughCircle() function of opencv which is used to highlight circle area within image. Here loop through contour to find all circles from left-right-top-down approach. This will save it in array where each index of array constitute four options/contours of bubble that means each row is saved in each index of array, hence getting a nested array.

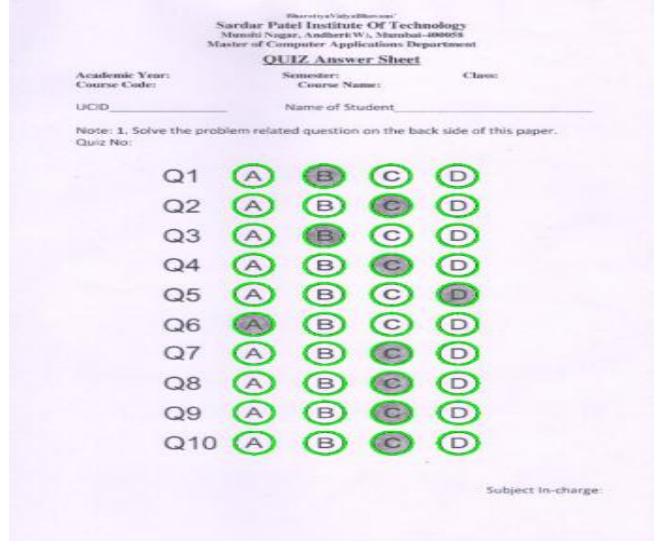

Fig. 4 Finding all bubbles

Step 10: Now next step is to perform function that check whether each answer highlighted is correct or not. Iinitially one has to provide array of our answer before. Now iterate through the contour array that was achieved earlier and compare each option with our initial answer array to check whether it is correct or not. But the initial step only found out the bubble contour, still one does not know which option is correct within them, After observing image of step 6 one can see the user highlighted or marker bubble option is white where as other options are black with white border. Again how to find this white bubble? this is done using thresh() of open cv using its parameter one can define whether following bubble is marker or not. If for first row actual answer is 3 then within contour array what needs to be done is select first option arrcontour[0][0] check whether it is white bubble using thresh if not move to arrcontour[0][1], In such way move to next option until one reach end of that row or complete nesting of inner array $[0,1,2,3]$ as there are have 4 options.

After that move to next index of bubble contour arrcontour[1][0] and again repeated previous step, for all index.

In case actual and thresh contour bubble match then increment a counter of correct array. Finally it has final number of correct options.

Here the correct answer is marked with green border where as wrong answer is marked with red border this can be done using drawContour() function of open cv.

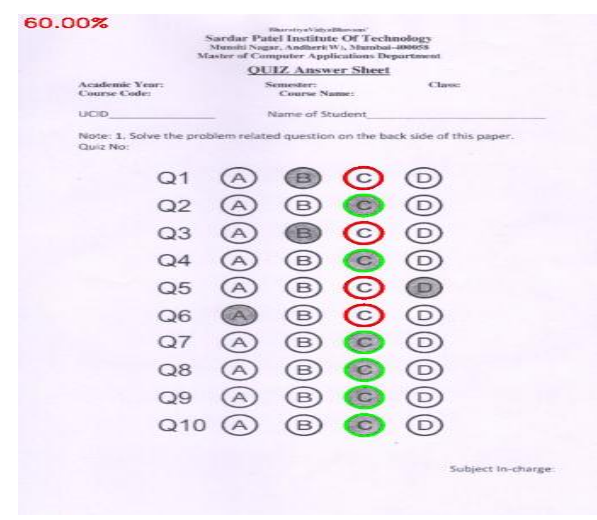

Fig. 5 Final score

Step 11: After reaching last contour of last row, then invoke a function which reads a excel from particular location and the enters marks (score present in correct counter) in it. Then apply basic formula to calculate percentage and display it. 


\section{FUTURE WORK}

Currently what the system is doing is that it take screenshot of answer sheet and then pass it to algorithm to perform it's grading. In future work, proposed system should act as real-time grading system, that means when paper is hold in front of webcam rather than taking screenshot the webcam will directly check the paper in real-time and display marks thus reducing implementation time, this will be achieve using using FLANN. Also later system will be integrating OMR with OCR so that in future the name, roll number and other text feature can be extracted. The plan is to use tesseract library to achieve this goal. Also currently the marks are getting stored at excel sheet, our future goal will be to send the marks obtained to relevant student automatically, this can be achieved by using Microsoft flow which is an automation tool that allows performing any sort of automation, here as each excel records get inserted particular event will be triggered and its corresponding action (send mark to user) will be invoked.

\section{ACKNOWLEDGMENT}

This research paper was supported by the Sardar Patel Institute of Technology. We thank our faculties who provided insight and expertise that greatly assisted the research. We would also like to thank all the other contributor available online who have made a deep impact in the field of opencv and OMR technology.

\section{REFERENCES}

[1] Digital Image Processing (3rd Edition) -Rafael C. Gonzalez

[2] Learning OpenCV-Computer Vision with the OpenCV Library- Gary Bradski, Adrian Kaehler

[3] High-Level Computer Vision Using OpenCVGraphics, Patterns and Images Tutorials (SIBGRAPI-T), 2011 24th SIBGRAPI Conference on 28-30 Aug. 2011

[4] OpenCV implementation optimized for a cell broadband engine processor- Sugano, H.; Dept. of Commun. \& Comput. Eng., Kyoto Univ., Kyoto; Miyamoto, R.

[5] B. Girod. "EE 368 Digital Image Processing Notes". EE 368 Digital Image Processing. Spring 2011-2012

[6] https://docs.opencv.org/2.4.13.7/doc/tutorials/features2d/fe ature_flann_matcher/feature_flann_matcher.html 